\title{
Principais cuidados desenvolvidos para pacientes em estado terminal: uma revisão integrativa da literatura
}

\author{
Main care developed for patients in terminal state: an integrative literature review \\ Principales cuidados desarrollados para pacientes terminales: una revisión integradora de la
}

\section{literatura}

Recebido: 30/01/2022 | Revisado: 09/02/2022 | Aceito: 21/02/2022 | Publicado: 03/03/2022

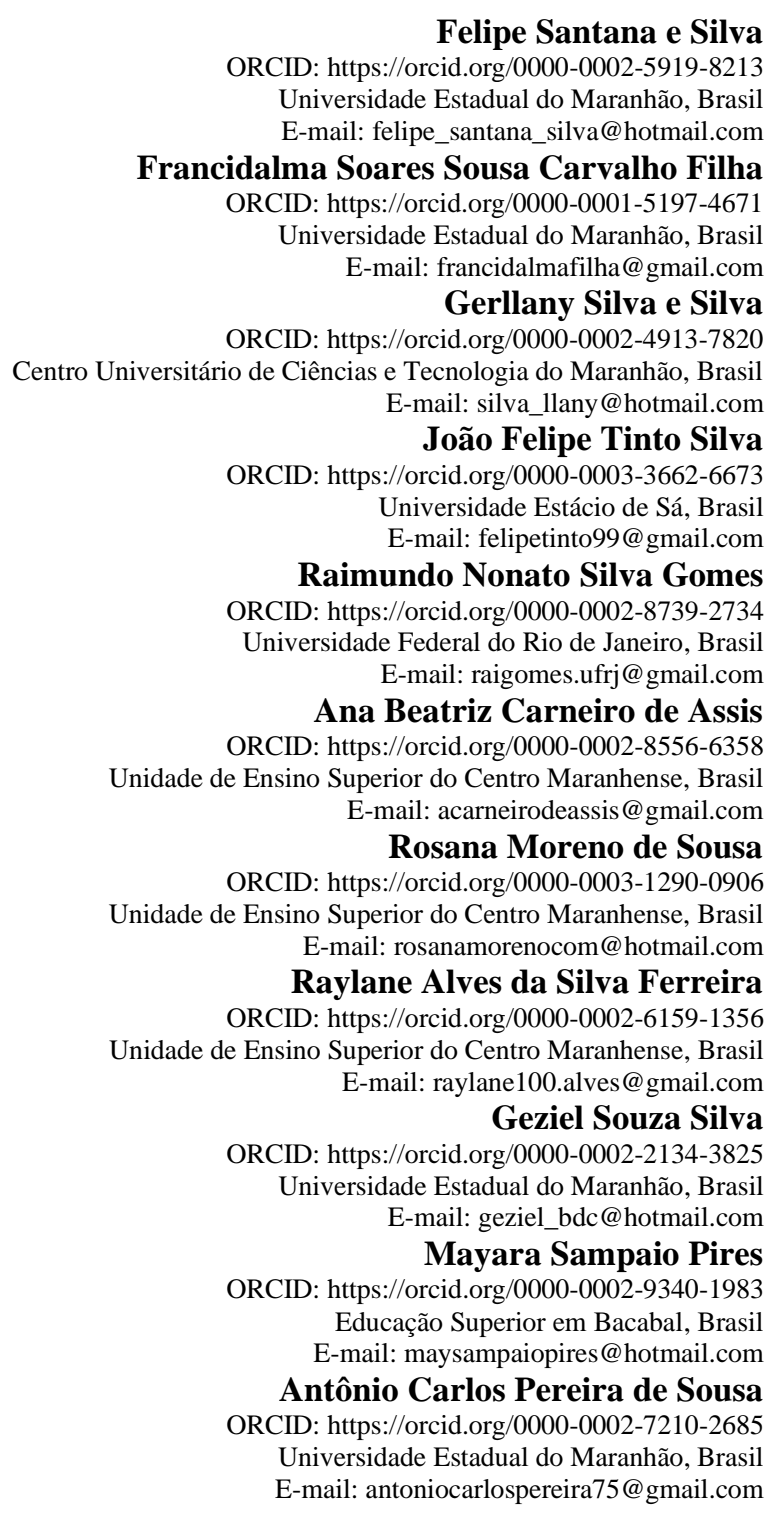

\section{Resumo}

Este estudo objetivou descrever com base na literatura os principais cuidados prestados a pacientes em estado terminal. Trata-se de uma revisão integrativa da literatura, para a busca foi utilizado os Descritores em Ciências da Saúde: Cuidado Paliativo; Profissionais da Saúde; Unidade de Terapia Intensiva; Doença terminal em português e inglês é foi realizada através das bases: Literatura Latino-Americana e do Caribe em Ciências da Saúde, Sistema Regional de Informação em Linha para Revistas Científicas da América Latina, Caribe, Espanha e Portugal, Grupo Verde de Agroecologia e Abelhas, Sistema Online de Busca e Análise de Literatura Médica. Desta forma após a busca sistemática e aplicação dos critérios de inclusão e exclusão foram analisados 09 artigos científicos no período de 2017 a 2021. Os manuscritos permitiram concluir que os principais cuidados desempenhados para os pacientes em terminalidade que se encontram em 
cuidados paliativos são: a prestação de cuidados básicos aos pacientes que dependem de competência técnico-científica da equipe; o uso de fitoterápicos com as plantas medicinais; o uso de novas tecnologias, desde que respeitem os preceitos éticos, sobretudo voltado para melhoria da qualidade de vida; e o cuidado espiritual que mesmo não sendo desempenhado pela equipe, este pode ser despendido a medida que o paciente tem alguma crença. Vale ressaltar que apesar dos achados, ainda existe um vácuo na literatura, no que diz respeito aos cuidados paliativos. Uma vez que cada manuscrito traz um cuidado diferente. Assim sendo, sugere-se que novos estudos sejam realizados e que estes tragam uma sistematização dos cuidados, o que irá facilitar a sua aplicação.

Palavras-chave: Cuidados paliativos; Profissionais da saúde; Unidade de Terapia Intensiva; Doença terminal; Cuidados paliativos.

\begin{abstract}
This study aimed to describe, based on the literature, the main care provided to terminally ill patients. This is an integrative literature review. The Health Sciences Descriptors were used for the search: Palliative Care; Health professionals; Intensive care unit; Terminal illness in Portuguese and English was carried out through the following bases: Latin American and Caribbean Literature on Health Sciences, Regional Online Information System for Scientific Journals from Latin America, the Caribbean, Spain and Portugal, Grupo Verde de Agroecologia e Abelhas , Online System of Search and Analysis of Medical Literature. In this way, after the systematic search and application of the inclusion and exclusion criteria, 09 scientific articles were analyzed in the period from 2017 to 2021. The manuscripts allowed us to conclude that the main care performed for terminally ill patients who are in palliative care are: the provision of of basic care to patients who depend on the technical-scientific competence of the team; the use of herbal medicines with medicinal plants; the use of new technologies, as long as they respect ethical precepts, especially aimed at improving the quality of life; and spiritual care that, even if not performed by the team, can be spent as the patient has some belief. It is worth mentioning that despite the findings, there is still a vacuum in the literature with regard to palliative care. Since each manuscript brings a different care. Therefore, it is suggested that new studies be carried out and that they bring a systematization of care, which will facilitate its application.
\end{abstract}

Keywords: Palliative care; Health professionals; Intensive Care Unit; Terminal illness.

\begin{abstract}
Resumen
Este estudio tuvo como objetivo describir, con base en la literatura, los principales cuidados prestados a los pacientes terminales. Se trata de una revisión integrativa de la literatura, para la búsqueda se utilizaron los Descriptores de Ciencias de la Salud: Palliative Care; Profesionales de la salud; Unidad de terapia intensiva; Enfermedad terminal en portugués e inglés se llevó a cabo a través de las siguientes bases: Literatura Latinoamericana y del Caribe en Ciencias de la Salud, Sistema Regional de Información en Línea para Revistas Científicas de América Latina, el Caribe, España y Portugal, Grupo Verde de Agroecologia e Abelhas, Sistema en Línea de Búsqueda y Análisis de la Literatura Médica. De esta forma, luego de la búsqueda sistemática y aplicación de los criterios de inclusión y exclusión, se analizaron 09 artículos científicos en el período de 2017 a 2021. Los manuscritos permitieron concluir que los principales cuidados realizados a los pacientes terminales que se encuentran en cuidados paliativos son: la provisión de cuidados básicos a los pacientes que dependen de la competencia técnico-científica del equipo; el uso de medicamentos a base de hierbas con plantas medicinales; el uso de las nuevas tecnologías, siempre que respeten los preceptos éticos, especialmente encaminados a mejorar la calidad de vida; y cuidado espiritual que, aun no siendo realizado por el equipo, puede ser gastado según el paciente tenga alguna creencia. Cabe mencionar que, a pesar de los hallazgos, aún existe un vacío en la literatura con respecto a los cuidados paliativos. Ya que cada manuscrito trae un cuidado diferente. Por lo tanto, se sugiere que se realicen nuevos estudios y que traigan una sistematización de la atención, lo que facilitará su aplicación.
\end{abstract}

Palabras clave: Cuidados paliativos; Profesionales de la salud; Unidad de Cuidados Intensivos; Enfermedad terminal.

\title{
1. Introdução
}

A terminalidade parece ser o eixo central do conceito em torno da qual se situam as consequências. É quando se esgotam as possibilidades de resgate das condições de saúde do paciente e a possibilidade de morte próxima parece inevitável e previsível. Assim o paciente se torna "irrecuperável" e caminha para a morte, sem que se consiga reverter este caminhar (Gutierrez,2001).

Diante do exposto cuidados paliativos, são definidos pela Organização Mundial de Saúde (OMS) como uma abordagem que melhora a qualidade de vida dos pacientes e familiares que enfrentam problemas relacionados à doenças que ameaçam a vida. Prevenindo e aliviando o sofrimento por meio da identificação precoce, avaliação e tratamentos corretos da dor e outros problemas, sejam de ordem física, psicossocial ou espiritual. Portanto estes cuidados tratam de estratégias terapêutica que envolvem uma equipe multidisciplinar (especialidades médicas, de enfermagem, psicologia, nutrição, 
fisioterapia, fonoaudiologia, serviço social, terapia ocupacional, farmácia e conselhos espirituais e religiosos) que os implementam (OMS, 2018).

Ademais os cuidados paliativos, são aqueles que não tem mais a finalidade de curar, tendo em vista que a doença já é progressiva, irreversível e não responde ao tratamento, mas, sim proporcionar qualidade de vida nos momentos finais. É ainda o cuidado através, da assistência que visa à qualidade de vida e manutenção da dignidade humana no decorrer da doença, na terminalidade da vida, na morte e no período de luto. Além disso é um direito do paciente e dever dos profissionais de saúde de prestar uma assistência integral e contínua ao seu humano (Capelas et al., 2016).

Os cuidados mencionados anteriormente devem ser implementados o mais precocemente possível, e associados a outras ações de ampliação da vida, como a prática dos tratamentos consolidados na literatura como a quimioterapia e a radioterapia. No mais, os cuidados paliativos devem ser promovidos por distintas categorias profissionais, onde toda equipe multiprofissional deve trabalhar em conjunto e deve ser destinado para a família, a criança, o idoso e os sujeitos em situação de terminalidade (Santos el al., 2019, Rodrigues et al., 2017).

No Brasil, os cuidados paliativos, começaram a ser implantado no Brasil no ano de 1983 no HCPA, logo após o lançamento do Estuado Support nos Estados Unidos no ano de 1995 que refletia positivamente em vários países. Assim sendo, no ano de 2002, foi publicada a portaria n ${ }^{\circ}$ 19/GM que instituiu em no art. $1^{\circ}$ no campo do Sistema Único de Saúde (SUS), o programa Nacional de Assistência à Dor e Cuidados Paliativos, estimulando a discussão acerca da temática e meios de assistência (Brasil, 2020).

No ano de 2018, o Brasil contava com 177 serviços de Cuidados Paliativos, como mostra o documento Análise Situacional e Recomendações da ANCP para Estruturação de Programas de Cuidados Paliativos no Brasil, no ano de 2019 já era mais de 190. Esse aumento que chegou a quase $8 \%$ merece comemoração, mas é insuficiente para colocar o país no grupo de nações com melhor nível de cobertura em Cuidados Paliativos (Dos Santos et al., 2020).

Para Franco et al., (2017) a enfermagem possui o papel de profissional responsável por humanizar a assistência, pois como enfermeiro, sua visão deve estar atenta as reais necessidades que o paciente apresenta, podendo identificá-la rapidamente, seja de forma verbal, ou não verbal, e suprindo-as da melhor maneira possível, e quando não lhe couber, tendo voz e auxílio de uma equipe multiprofissional.

Assim sendo, segundo a nova resolução n 564/2017 é dever do enfermeiro, prestar assistência aos pacientes graves com risco de vida, assim como cuidados de enfermagem de maior complexidade técnica e que exijam conhecimentos científicos adequados, possuindo ainda a capacidade de tomar decisões imediatas. Além de correr o risco de ser punido, se por algum motivo causar qualquer dano ao paciente ou se omitir em planejar, agindo com as precauções adequadas, sendo estes danos enquadrados como infrações leves, graves ou gravíssimas (COFEN, 2017).

Diante disto, este estudo objetivou descrever com base na literata os principais cuidados prestados a pacientes em estado terminal.

\section{Metodologia}

Trata-se de uma revisão integrativa da literatura, que é um método de pesquisa da Prática Baseada em Evidência. Desta forma, esta revisão responde a uma ou mais perguntas e utiliza métodos explícitos para identificar, selecionar e avaliar criticamente os estudos (Mendes et al., 2008).

A pesquisa trilhou o seguinte percurso metodológico: Identificação do problema; busca na literatura; Extração dos dados dos estudos; Avaliação dos estudos; Interpretação dos resultados; Síntese dos resultados; súmula do conhecimento evidenciado. A estratégia de busca eletrônica se deu nas seguintes bases de dados: Literatura Latino-Americana e do Caribe em Ciências da Saúde (LILACS), Sistema Regional de Informação em Linha para Revistas Científicas da América Latina, Caribe, Espanha e 
Portugal (Latindex), Grupo Verde de Agroecologia e Abelhas (GVAA), Sistema Online de Busca e Análise de Literatura Médica (Medline)/PubMed, empregando, de forma isolada ou em combinação com a expressão boleana "And”, os Descritores em Ciências da Saúde (DeCS) e o Medical Subject Headings (MesH): Cuidados Paliativos (Palliative Care); Unidade de Terapia Intensiva (Intensive Care Units); Doença Terminal (Critical IIIness) (Whittemore; Knafl, 2005).

A seleção dos estudos foi realizada seguindo as diretrizes da Preferred Reporting Items for Systematic Reviews and Meta-Analyses. Os critérios de inclusão foram: produções científicas integrais em português e inglês disponíveis nas bases de dados selecionadas, no período de 2015 a 2021 e que tinham como objetivo a identificar os principais cuidados desempenhados pela equipe de enfermagem para pacientes em cuidados paliativos. Optou-se pela exclusão dos artigos de revisão da literatura e estudo de caso, textos incompletos, teses e dissertações, pois apresentam limitações para responder às questões norteadoras propostas pelos autores.

Para extração dos dados dos artigos, elaborou-se um instrumento contendo as seguintes informações: título, autores, periódico, ano de publicação, tipo de pesquisa, base de dados. A análise dos estudos encontrados foi feita de forma descritiva onde os mesmos foram analisados quanto ao ano, autoria, local do estudo, tipo de estudo, população-alvo, delineamento do estudo (Liberati et al., 2009).

\section{Resultados e Discussão}

Foram identificados 202 estudos nas bases de dados. Após eliminação de 42 artigos duplicados, foram selecionados 160 artigos. Desses, 34 foram excluídos após a análise dos títulos e resumos. Dos 126 artigos elegíveis, foram excluídos pelos seguintes motivos: 28 não estavam disponíveis na integra; 33 não atendiam ao recorte temporal; 25 não avaliavam os principais cuidados de enfermagem prestados a cuidados de enfermagem prestados a pacientes em estado terminal, e 29 estudos eram de revisão. Ao final, 09 estudos foram inclusos na revisão integrativa compondo a amostra. Não foram encontrados estudos por meio da busca manual nas referências dos artigos encontrados.

A Figura 1 apresenta a síntese do processo de seleção dos artigos. 
Figura 1 - Fluxograma de identificação e seleção dos artigos segundo diretrizes

do instrumento PRISMA, 2015 á 2021.

IDENTIFICAÇ̃̃o

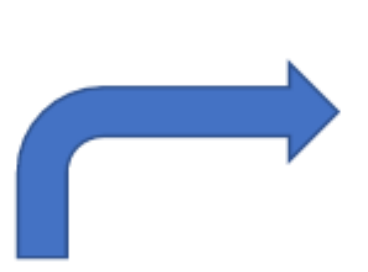

\begin{tabular}{|c|}
\hline Busca nas Bases \\
eletrônicas $(\mathrm{n}=202)$ \\
LILACS $(\mathrm{n}=72)$ \\
Medline/PubMed $(\mathrm{n}=56)$ \\
LATINDEX $(\mathrm{n}=48)$ \\
GVAA $(\mathrm{n}=26)$ \\
\hline
\end{tabular}

Artigos excluídos em duplicidade $(n=42)$

Artigos excluídos após leitura do título e resumo $(n=34)$
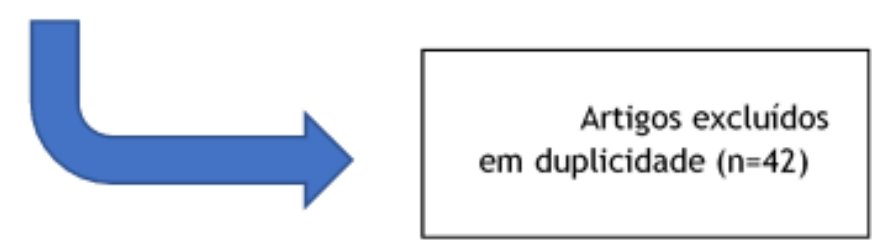
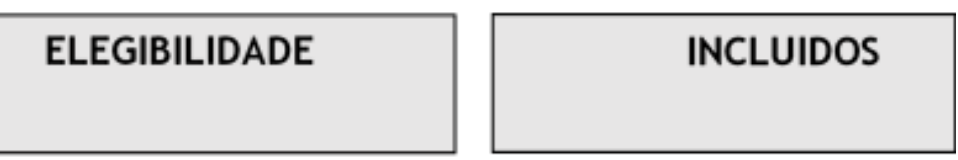

Artigos incluídos na revisão $(n=09)$

Fonte: Autores. 
Dos estudos analisados, quanto às características gerais, 100\% (n=9) foram escritos em Língua Portuguesa, com abordagem qualitativa $66,6 \%(n=6)$ e $88,8 \%(n=8)$ trataram-se de pesquisas realizadas no Brasil. O último dado mencionado, demonstra que os pesquisadores brasileiros nos últimos anos buscaram, se aprofundar nesta área de conhecimento, trazendo assim grandes benefícios para os pacientes, que necessitam destes cuidados.

No que se refere aos objetivos propostos pelos estudos, independentemente da diversidade entre eles, constatou-se que $88,88 \%(\mathrm{n}=8)$, analisaram o conhecimento dos profissionais, sejam estes nos mais diversos setores, além de caracterizar as equipes e o nível de conhecimento destes em relação aos cuidados paliativos. Já somente 11,11\% (n=1) buscou comparar o alívio dos sintomas obtido por uma equipe interconsultora em cuidados Paliativos (ICP) ao obtido por equipe de cuidado tradicional (CT), em pessoas em cuidados paliativos.

Quadro 1. Síntese dos estudos incluídos na revisão integrativa, nas bases LILACS, LATINDEX, MEDLINE/PubMed, GVAA, no período de 2017 a 2020. CAXIAS - MA, 2021. (n=9).

\begin{tabular}{|c|c|c|c|c|c|}
\hline $\mathbf{N}$ & TITULO & AUTORES & PERIÓDICO & ANO & $\begin{array}{l}\text { ABORDAGEM/TIPO E } \\
\text { LOCAL DO ESTUDO }\end{array}$ \\
\hline $\mathbf{I}$ & $\begin{array}{l}\text { Enfermagem e cuidados paliativos, } \\
\text { reconhecendo valores }\end{array}$ & $\begin{array}{l}\text { SANTIAGO, E.J.P; ALVES, N.C.C; } \\
\text { FREIRE, A.K.S; KORINFSKY, J,P }\end{array}$ & $\begin{array}{l}\text { Revista Saúde e } \\
\text { Desenvolvimento }\end{array}$ & 2017 & $\begin{array}{l}\text { Quantitativa; Descritiva; } \\
\text { Exploratória } \\
\text { (Brasil) }\end{array}$ \\
\hline II & $\begin{array}{l}\text { Permissão de partida: um cuidado } \\
\text { espiritual de enfermagem na } \\
\text { finitude humana }\end{array}$ & $\begin{array}{l}\text { ZENEVICZ, L.T; BITENCOURT, } \\
\text { J.V.O.V; LÉO, M.M.F; } \\
\text { MADUREIRA, V.S.F; THOFEHRN, } \\
\text { M.B; CONCEIÇÃO, V.M }\end{array}$ & $\begin{array}{l}\text { Revista Brasileira de } \\
\text { Enfermagem }\end{array}$ & 2020 & $\begin{array}{l}\text { Qualitativa; Reflexiva } \\
\text { (Brasil) }\end{array}$ \\
\hline III & $\begin{array}{l}\text { Atuação da equipe de enfermagem } \\
\text { em cuidados paliativos }\end{array}$ & COSTA, M.B; SILVA, D.A & $\begin{array}{l}\text { Research, Society } \\
\text { and Development }\end{array}$ & 2021 & $\begin{array}{c}\text { Qualitativa; Observacional; } \\
\text { Transversal. } \\
\text { (Brasil) }\end{array}$ \\
\hline IV & $\begin{array}{c}\text { Cuidados paliativos e limitação de } \\
\text { suporte de vida em terapia } \\
\text { intensiva }\end{array}$ & $\begin{array}{l}\text { PEGORARO, M.M.O; PAGANINI, } \\
\text { M.C }\end{array}$ & Revista Bioética & 2019 & $\begin{array}{l}\text { Qualitativa; Descritiva } \\
\text { (Brasil) }\end{array}$ \\
\hline $\mathbf{V}$ & $\begin{array}{l}\text { Cuidados paliativos em unidade de } \\
\text { terapia intensiva: percepções dos } \\
\text { profissionais de enfermagem }\end{array}$ & $\begin{array}{l}\text { FARIA, T.N.T; CARBOGIM, F.C; } \\
\text { ALVES, K.R; TOLEDO, L.V; } \\
\text { MARQUES, D.A }\end{array}$ & $\begin{array}{c}\text { Revista de } \\
\text { Enfermagem da Ufpe }\end{array}$ & 2017 & $\begin{array}{l}\text { Qualitativa; Descritiva } \\
\text { (Brasil) }\end{array}$ \\
\hline VI & $\begin{array}{l}\text { Plantas medicinais utilizadas na } \\
\text { auto atenção por pessoas com } \\
\text { câncer em cuidado paliativo }\end{array}$ & $\begin{array}{c}\text { BONOW, C.T; CEOLIN T; LOPES, } \\
\text { C.V; ZILLMER, J.G.V; VARGAS, } \\
\text { N.T.C; HECK, R.M. }\end{array}$ & $\begin{array}{l}\text { Texto e Contexto } \\
\text { Enfermagem }\end{array}$ & 2020 & $\begin{array}{c}\text { Qualitativa; Exploratório; } \\
\text { Descritivo } \\
\text { (Brasil) }\end{array}$ \\
\hline VII & $\begin{array}{l}\text { Validação do resultado controle dos } \\
\text { sintomas para pacientes com } \\
\text { insuficiência cardíaca em cuidados } \\
\text { paliativos }\end{array}$ & $\begin{array}{l}\text { AFONSO, B.Q; FERREIRA, A.C; } \\
\text { BUTCHER, R.C.G.S }\end{array}$ & $\begin{array}{l}\text { Revista Gaúcha de } \\
\text { Enfermagem }\end{array}$ & 2020 & $\begin{array}{l}\text { Quantitativa; Descritivo } \\
\text { (Brasil) }\end{array}$ \\
\hline VIII & $\begin{array}{c}\text { Equipe interconsultora em cuidados } \\
\text { paliativos: alívio de sintomas nas } \\
\text { primeiras } 48 \text { horas de } \\
\text { hospitalização }\end{array}$ & $\begin{array}{l}\text { SILVA, M.A.S; DINIZ, M.A; } \\
\text { CARVALHO, R.T; CHIBA, T; } \\
\text { PIMENTA-MATTOS, C.A }\end{array}$ & $\begin{array}{l}\text { Revista Brasileira de } \\
\text { Enfermagem }\end{array}$ & 2020 & $\begin{array}{l}\text { Quantitativa; } \\
\text { Descritiva } \\
\text { (Brasil) }\end{array}$ \\
\hline IX & $\begin{array}{c}\text { Percepção dos profissionais de } \\
\text { saúde sobre os cuidados paliativos } \\
\text { neonatais }\end{array}$ & $\begin{array}{l}\text { SILVA, E.M.B; SILVA, M.J.M; } \\
\text { SILVA, D.M }\end{array}$ & $\begin{array}{l}\text { Revista Brasileira de } \\
\text { Enfermagem }\end{array}$ & 2019 & $\begin{array}{l}\text { Qualitativa; Fenomenológic: } \\
\text { (Portugal) }\end{array}$ \\
\hline
\end{tabular}

Fonte: Silva (2021).

$\mathrm{Na}$ análise do conteúdo dos estudos, constatou-se que existem inúmeros tipos de cuidados desenvolvidos pela equipe de saúde para como os pacientes em estado terminal. Ficou evidente que boa parte dos estudos se preocupou em analisar o conhecimento destes profissionais sobre estes tipos de cuidados, que visam em sua grande maioria amenizar o "sofrimento" destes pacientes. Os seguintes aspectos deixam isso mais explicitado foram: 
No primeiro estudo analisado, os autores concluíram que o conhecimento da equipe no total sobre a temática ainda é insatisfatório. Pois, em alguns ambientes estes cuidados não podem ser desempenhados, logo a observância de quais pacientes podem ser incluídos nos mesmo e de fundamental importância para uma assistência de qualidade logo o conhecimento destes profissionais mostrou-se razoável. Porém, sobre o conceito, ou seja, os componentes essenciais, como procedimentos tecnológicos prioritários a serem desempenhados, o conhecimento foi deficitário. Assim sendo, o conhecimento da equipe dos setores do hospital supracitado ainda é insatisfatório, o que pode interferir significativamente, no cuidado paliativo ofertado pela mesma (Santiago et al., 2017).

Já no segundo estudo analisado, os estudiosos constataram que a permissão de partida é um cuidado espiritual que facilita uma relação terapêutica intencional de confiança e segurança entre o profissional, o paciente e a família, que isso propiciaria a expressão de sentimentos, crenças e rituais religiosos ou espirituais que auxiliam na situação de morte ou morrer. Desta forma, cunhou-se um conceito estruturado por palavras e atitudes reforçando o positivismo, buscando um estado de consciência de paz é promoção da dignidade no processo de morte e morrer, bem como um tempo oportuno para que o paciente, a família e a equipe possam vivenciar o recolhimento e despedida (Zenevicz et al., 2020).

Ademais em outro manuscrito, a pesquisa possibilitou aos autores compreender a dificuldade dos profissionais que atuam como os pacientes em estado terminal. Onde alguns profissionais mostraram certa falta de conhecimento tanto teórico quanto prático sobre a assistência necessária para dispensar este tipo de cuidado. Além da falta de conhecimento por parte de alguns, outros apresentaram uma forte sensibilidade em relação a temática. Apensar de saber que a morte se faz presente no diaainda destes profissionais, muitos não se consideram preparados para este momento (Costa \& Silva, 2021).

No estudo de Pegoraro e Paganini (2019), o mesmo possibilitou aproximar pesquisadores e membros da equipe multidisciplinar da Unidade de Cuidados Intensivos (UTI), onde os profissionais reconheceram a necessidade de estabelecer critérios para atender paciente com limitação de suporte de vida em UTI e a importância dos cuidados paliativos, sendo possível aplicá-los por meio de ações sistematizadas. Assim sendo, o estudo permitiu concluir que uma pequena amostra da grande complexidade existente entre as duas concepções ainda em construção no cenário brasileiro, que podem usufruir da bioética, como campo do saber, para aprimorar as tomadas de decisão. Onde de um lado se tem o conceito de cuidado intensivo, vestido de tecnologia e busca incessante pela recuperação da vida e outrem tem-se cuidado paliativo, voltado para a manutenção da qualidade de vida.

Em interim uma pesquisa realizada no estado de Minas Gerais, no qual os seus resultados demonstraram que os cuidados paliativos em UTI consistem, em prestar uma assistência básica ao paciente, valendo-se de competências técnico-científicas aliadas a comportamentos e atitudes específicas. Os autores ressaltam ainda que apesar da sua evidente importância, o cuidado paliativo ainda não é conhecido de forma uniforme, o que revela a fragilidade no processo de formação e treinamento profissional, e que na grande maioria das vezes a família assume papel fundamental no enfrentamento da terminalidade, sendo uma aliada ao tratamento no processo de cuidado (Faria et al., 2017).

Na pesquisa de Bonow et al., (2020), foi possível identificaram que as pessoas com câncer em cuidado paliativo já utilizavam antes do adoecimento e continuaram fazendo o uso de plantas medicinais em busca de uma ação terapêutica, tanto para amenizar os sintomas causados pelo estágio final da doença, quanto para a cura do câncer. E que o conhecimento das plantas medicinais, na maioria das vezes, foi passado de geração em geração, ou por amigos, e, geralmente, o uso destas não são informadas aos profissionais de saúde.

Em seguida a pesquisa de Afonso et al., (2020) contribuiu para a prática assistencial pois permitiu uma avaliação multidimensional do controle de sintomas, o que é um avanço em termos de outros instrumentos disponíveis na literatura. No campo da pesquisa de enfermagem os achados são importantes porque fornecem uma estimativa de características válidas para avaliar o controle dos sintomas no cenário de cuidados paliativos a paciente com Insuficiência cardíaca (IC). 
Outrossim em um estudo realizado em São Paulo, os dados demonstraram a dificuldade para o alívio de sintomas de modo rápido e clinicamente relevante em doentes oncológicos em cuidados paliativos, e a necessidade de aperfeiçoar o atendimento a esses doentes. Porém ressalta-se a necessidade de novas investigações que possam responder em que condições e unidades de cuidados os doentes obtém melhor controle do seu sofrimento (Silva et al., 2020).

Não obstante no último artigo analisado, os autores constaram que apesar da falta de formação em cuidados paliativos, os profissionais revelaram preocupação com a dignidade, qualidade de vida e conforto dos recém-nascidos e família. Expressando assim as dificuldades emocionais e relacionais nos acompanhamentos das trajetórias de doenças graves e mortes a nível da decisão ética no findar da vida. Ainda os estudiosos salientam que os profissionais são sensíveis à dor e sofrimento e mostram-se dedicados e comprometidos no cuidar dos recém-nascidos e da família (Silva et al., 2019).

Como limitação vale ressaltar que apesar dos achados, ainda existe um vácuo na literatura, no que diz respeito aos cuidados paliativos. Uma vez que cada manuscrito traz um cuidado diferenciado. Assim sendo, sugere-se que novos estudos sejam realizados e que estes tragam uma sistematização dos cuidados, o que irá facilitar a aplicação dos mesmos por estes profissionais que lidam com a terminalidade da vida.

\section{Conclusão}

Os manuscritos permitiram concluir que os principais cuidados desempenhas para os pacientes em terminalidade que se encontram em cuidados paliativos são: a prestação de cuidados básicos aos pacientes que dependem de competência técnicocientífica da equipe; o uso de fitoterápicos com as plantas medicinais (que geralmente seu uso começa antes mesmo deles entrarem no último estado patológico e permaneçam até estágio paliativo); o uso de novas tecnologias, desde que respeitem os preceitos éticos, sobretudo voltado para melhoria da qualidade de vida; e o cuidado espiritual que mesmo não sendo desempenhado pela equipe, este pode ser despendido a medida que o paciente tem alguma crença.

Ademais nesta revisão integrativa, identificou-se que existem inúmeros tipos de cuidados despendidos para os pacientes em estado terminal. Ficou claro também nesta análise que a grande maioria dos profissionais que lidam com esses pacientes, desconhecem estes cuidados e aqueles que conhecem não sabem como operacionalizá-los, logo a falta de treinamento é a principal causa da não aplicabilidade dos cuidados, uma vez que a grande maioria dos profissionais demonstraram interesse em desenvolvê-las.

\section{Referências}

Afondo, B. Q., Ferreira, N. C., \& Butcher, R. C. G. S. (2020). Validação do resultado controle dos sintomas para pacientes com insuficiência cardíaca em cuidados paliativos. Revista Gaúcha de Enfermagem, 41(spl), 1-8.

Bonow, C. T., Ceolin, T., Lopes, C. V., Zillmer, J. G. V., Vargas, N. R. C., \& Heck, R. M. (2020). Plantas medicinais utilizadas na autoatenção por pessoas com câncer em cuidado paliativo. Texto \& Contexto Enfermagem, 29(spl), 1-16.

Brasil. Ministério da Saúde. Manual de Cuidados Paliativos. (2020). < https://cuidadospaliativos.org/uploads/2020/12/Manual-Cuidados-Paliativos.pdf >.

Capelas, M. L., Da Silva, S. C. F. S., Alvarenga, M. I. S. F., \& Coelho, S. P. (2016). Cuidados paliativos: O que é importante saber. Patient. Care, 16-20.

COFEN. Conselho Federal de Enfermagem. (2017). Resolução COFEN $n^{\circ}$ 564/2017. Novo Código de Ética dos Profissionais de Enfermagem. http://www.cofen.gov.br/resolucao-cofen-no-5642017_59145.html.

Costa, B. M., \& Silva, D. A. (2021). Atuação da equipe de enfermagem em cuidados paliativos. Research, Society and Development. 10(2), 1-16.

Dos Santos, A. F. J., Ferreira, E. A. L., \& Guirro, U. B. P. (2020). Atlas dos Cuidados Paliativos no Brasil 2019 (1. ed.). São Paulo, SP: Academia Nacional de Cuidados Paliativos - ANCP.

Faria, T. N. T., Carbogim, F. C., Alves K. R., Toledo, L. V., \& Marques, D. A. (2017). Cuidados Paliativos em Unidade de Terapia Intensiva: percepções dos profissionais de enfermagem. Revista de Enfermagem da UFPE. 11(5), 1996-2002.

Franco, H. C. P., Stigar, R., Souza, S. J. P., \& Burci, L. M. (2017). Papel da enfermagem na equipe de cuidados paliativos: a humanização no processo da morte e morrer. Revista Gestão \& Saúde. 17(2), 48-61. 
Research, Society and Development, v. 11, n. 3, e44011326324, 2022

(CC BY 4.0) | ISSN 2525-3409 | DOI: http://dx.doi.org/10.33448/rsd-v11i3.26324

Hermes, H. R., \& Lamarca, I. C. A. (2013). Cuidados paliativos: uma abordagem a partir das categorias profissionais de saúde. Ciência \& Saúde Coletiva. 18(9), $2577-2588$

Matos, E., Pires, D. E. P., \& Gelbcke, F. L. (2012). Implicações da interdisciplinaridade na organização do trabalho da enfermagem: estudo em equipe de cuidados paliativos. Revista Eletrônica de Enfermagem. 14(2), 230-239.

Mendes, K. D. S., Silveira, R. C. C., \& Galvão, C. M. (2008). Revisão integrativa: método de pesquisa para a incorporação de evidências na saúde e na enfermagem. Texto contexto - enferm on line. 17(4), 758-764.

OMS. Organização Mundial de Saúde. (2018). Cuidados paliativos. Organización Mundial de la Salud [Internet]. Notas descriptivas. https://bit.ly/2LJnLND. Acesso em: 26 de julho de 2021.

Pegoraro, M. M., \& Paganini, M. C. (2019). Cuidados paliativos e limitação de suporte de vida em terapia intesiva. Revista Bioética. 27(4), 699-710.

Rodrigues, G. G. F., Duarte, M. C. S., Mamede, R. S., Simões, K. M., Santos, J. S., \& Oliveira, T. C. (2017). Cuidados Paliativos Direcionados ao cliente Oncológico: estudo bibliométrico. Rev enferm UFPE on line. 11(3), 1349-1656.

Santiago, E. J. P., Alves, N. C., Freire A. K. S., \& Korinfsky, J. P. (2017). Enfermagem e cuidados paliativos, reconhecendo valores. Revista Saúde e Desenvolvimento. 11(9), 318-339.

Dos Santos, R. B., Gomes, M. C., Bonadio, C. B., Ferreira, P. S., Bartequini, R. B \& Rodrigues, L. F. (2019). Estudo Observacional Restrospectivo sobre o Perfil de pacientes que receberam terapia de Sedação Paliativa em Unidade de Cuidados Paliativos de Hospital de Câncer no Brasil. Revista Brasileira de Cancerologia. 65(1), 1-7.

Gutierrez, P. L. O que é o paciente terminal? (2001). Revista da Associação Médica Brasileira. 47(2),92. <https://doi.org/10.1590/S0104-42302001000200010>.

Silva, E. M. B., Silva., M. J. M., \& Silva, D. M. (2019). Percepção dos profissionais de saúde sobre os cuidados paliativos neonatais. Revista Brasileira de Enfermagem. 72(6), 1787-1794.

Silva, M. A. S., Diniz, M. A., Carvalho, R. T., Chiba, T., \& MatoS-Pimenta, C. A. (2020). Equipe interconsultora em cuidados paliativos: alívio de sintomas nas primeiras 48 horas de hospitalização. Revista Brasileira de Enfermagem. 73(6), 1-8, 2020.

Whittemore, R., \& Knafl, K. (2005). The integrative review: updated methodology. J Adv Nurs on line, Inglaterra. 52(5), 546-553.

Zenevicz, L. T., Bitencourt, J. V. O. V., Léo, M. M. F., Madureira, V. S. F., Thofehrn, M. B., \& Conceição, V. M. (2020). Permissão de partida: um cuidado espiritual de enfermagem na finitude humana. Revista Brasileira de Enfermagem. 73(3), 1-5. 